\title{
Desarrollo de un sistema de gestión energética en la Clínica Portoazul
}

\section{Development of an energy management system at the Portoazul Clinic}

Fecha de Recibido: 20/01/2021 Fecha de Aceptado: 30/03/2021

\author{
Sebastián Andrés Botero Ebrat \\ Clínica Portoazul. Barranquilla (Colombia) \\ sebastianbotero@gmail.com
}

Para citar este artículo:

S. Botero, "Desarrollo de un sistema de gestión energética en la Clínica Portoazul", LADEE, vol. 2, no. 1, pp. 1-11, 2021.

Resumen - La evaluación del desempeño energético de edificaciones hospitalarias es un tema poco discutido en la literatura. En general, los estudios disponibles se enfocan en la introducción de nuevas tecnologías y complejos métodos de predicción del consumo energético, y sus resultados se orientan fundamentalmente a los sistemas de calefacción. En este trabajo se desarrolla un sistema de gestión energética, basado en la norma ISO 50001, para la Clínica Portoazul, ubicada en la primera zona franca de salud de la Costa Atlántica colombiana. Durante el estudio se identificó la electricidad como el principal portador energético consumido en la clínica. Adicionalmente, se identificaron los equipos y/o sistemas con mayor demanda eléctrica. Basado en las diferentes mediciones y datos de consumo eléctrico de la clínica, se desarrolló una línea base y un indicador de desempeño garantizando una correlación $\mathrm{R}^{2}>0.6$. El indicador de desempeño se utilizó como base para desarrollar el sistema de gestión energética que evalúa diariamente el desempeño de la clínica. Los resultados de implementación del sistema de gestión entre enero y febrero de 2020 muestran una reducción del consumo de electricidad diario entre un 1 y un $15 \%$ respecto a la línea base. Este sistema es un enfoque más simple, práctico y económico que los discutidos en la literatura.

Palabras clave- Eficiencia energética; gestión energética; indicador de desempeño energético; línea base; edificaciones hospitalarias
Abstract- Assessing the energy performance of hospital building is little discussed in literature. Overall, the available studies focus on the use of new technologies, complex models to predict the electricity consumption, mostly addressing saving measures for heating systems. This study develops an ISO 50001 based energy management system for the Clinic Portoazul, located in the first health free zone of the Colombian Atlantic Coast. During the study, electricity was identified as the main energy carrier used in the clinic. Furthermore, the equipment and health systems with the highest consumption of electricity were identified. Based on the measured and historic data, an energy performance indicator was developed with a correlation of $\mathrm{R}^{2}>$ 0.6 . The energy management system was based on the indicator. Results from the energy management system between in January and February of 2020 show a reduction of the daily electricity demand between 1 and $15 \%$ as compared to the baseline. This system is a more practical and simple approach to address energy management in hospital facilities contrasted to other approaches.

Keywords- Energy efficiency; energy management; energy performance indicator; baseline; hospital facilities 


\section{INTRODUCCION}

El aumento sistemático de la población mundial, principal impulsor del incremento en la demanda de energía y del desempeño ambiental en la actualidad, generan la necesidad de incrementar la eficiencia en el consumo de energía. En particular, las edificaciones y la construcción representan alrededor del 36\% del consumo de energía global, contribuyendo con el $39 \%$ de las emisiones de $\mathrm{CO}_{2}$ [1]. En un contexto de crecimiento económico acelerado, las edificaciones están emergiendo como un sector crítico en el consumo de energía, consumiendo entre el 30 y el 40\% del consumo eléctrico en horarios de demanda pico [2]. Considerando que en promedio pasamos el $90 \%$ del tiempo en edificaciones, es necesario reducir el consumo de energía mediante mejores estándares de eficiencia [3]. Para esto es necesario transformar el enfoque energético actual en las edificaciones y evolucionar a procesos más eficientes y amigables con el medioambiente [3]. Específicamente, los hospitales representan el 6\% del consumo de energía de las ediciones, fundamentalmente para la Calefacción, Ventilación y Acondicionamiento de Aire (HVAC) [4]. Por características como el servicio de $24 \mathrm{~h}$ y el uso de equipos energéticamente intensivos para servicios ofrecer servicios médicos, las edificaciones hospitalarias son grandes consumidoras de energía con gran potencial de ahorro [5]-[8]. En general, las instalaciones hospitalarias tienen mayores demandas de ventilación, aire acondicionado, y esterilidad, lo que se traduce en mayores consumos de energía.

Se han desarrollado pocos estudios para evaluar el despeño energético en edificaciones hospitalarias. En Nápoles (Italia), por ejemplo, se ha desarrollado un análisis de reacondicionamiento energético en 4 edificaciones de un hospital universitario [9]; en general, se consideró como medidas de ahorro energético el uso de tecnologías y estrategias enfocadas a la calefacción, definidas a partir de simulaciones dinámicas del desempeño energético y campañas experimentales basadas en diseño de experimentos para evaluar la influencia de variables como la humedad y la temperatura en el consumo energético; y los resultados señalan un potencial significativo de eficiencia energética. Por otra parte, también se creó un método para establecer una línea de base comparación para edificaciones hospitalarias y definir los factores que afectan su consumo energético [5]; el estudio se desarrolló en 45 grandes hospitales (32\% del total) en Tailandia; y como resultado se definió una línea base de comparación entre hospitales grandes que puede servir para el desarrollo de planes de eficiencia energética en hospitales. Otro estudio, enfocado en la reducción de la emisión de gases de efecto invernadero, evaluó el potencial de ahorro en dos edificaciones hospitalarias construidas entre 1970 y 1980 en la Europa del este [10]; se logró reducir el consumo energético y las emisiones de gases de efecto invernadero transformando los hospitales en edificaciones inteligentes. En Marruecos se dieron avances en una guía de buenas prácticas para mejorar la eficiencia energética en edificaciones hospitalarias para contribuir a los objetivos nacionales de eficiencia energética [11]; la metodología analiza diferentes EnPIs y su aplicación en contextos similares; y los resultados señalan que, en instalaciones hospitalarias, la introducción de estrategias de eficiencia energética está en sus inicios. Por otra parte, en un hospital en Shanghái (China), se validó el uso de modelos de aprendizaje automático para predecir el consumo energético en edificaciones hospitalarias [7]; sus resultados muestran como el uso de datos diarios del consumo energético permite predecir de forma más precisa el consumo energético que los datos semanales; adicional, se identificaron la temperatura ambiente, la presión atmosférica y la operación del sistema de aire acondicionado como los principales factores que afectan el consumo energético en edificaciones hospitalarias. Finalmente, el desempeño térmico de las oficinas médicas en una edificación hospitalaria en Lisboa (Portugal) se evalúo con un estudio experimental holístico basado en métodos numéricos [6]; en este caso se monitoreó la temperatura, los flujos de calor y la irradiación en 2 oficinas médicas durante temporadas de calentamiento y enfriamiento; como resultado se recomendó el uso de ventanas de cristal reflectivo doble para reducir el consumo de energía en el acondicionamiento de aire.

En general, el número de estudios enfocados a la evaluación energética de edificaciones hospitalarias es limitado, y fundamentalmente se han enfocado a reducir el consumo energético de la calefacción, o al uso de tecnologías y sistemas complejos para evaluar y controlar el desempeño energético. 
En Colombia, el plan de acción indicativo de eficiencia energética, apunta entre otras medidas para edificaciones, a la actualización y consolidación de una línea base de consumo de energía [12].

La Clínica Portoazul, cuenta con sistema BASS (Building Automation and Security System) para la operación automatizada de los sistemas HVAC, iluminación, plantas eléctricas, subestación eléctrica, ascensores, gases medicinales, extinción y detección de incendios, CCTV y control de acceso. A pesar de esto, no cuenta con un sistema adecuado de monitoreo de la eficiencia global de la clínica. Conociendo que, sin un monitoreo adecuado no es posible garantizar estándares de eficiencia alto, se propone el desarrollo de un sistema de gestión energética, para identificar y corregir ineficiencias durante la explotación de los sistemas energéticos, y en el uso de los diferentes portadores energéticos.

Este artículo se enfoca en el trabajo desarrollado para generar una metodología de gestión energética para la Clínica Portoazul, la cual no implique el uso de tecnologías y sistemas sofisticados para el monitoreo y control del consumo de energía, y permita mejorar su desempeño energético.

\section{Metodología}

La evaluación energética de edificaciones permite una compresión de las características del consumo energético y su potencial de ahorro [13]. La ISO 50001 establece un marco de gestión de la energía para instalaciones industriales, comerciales o institucionales, desde su adquisición hasta su uso [21] (Fig. 1). A largo plazo, se espera que la aplicación de esta norma contribuya a mejoras de más del $20 \%$ de la eficiencia energética en instalaciones industriales, comerciales e institucionales [14], [15]. Esta es una herramienta esencial para el desarrollo de sistemas de gestión energética.

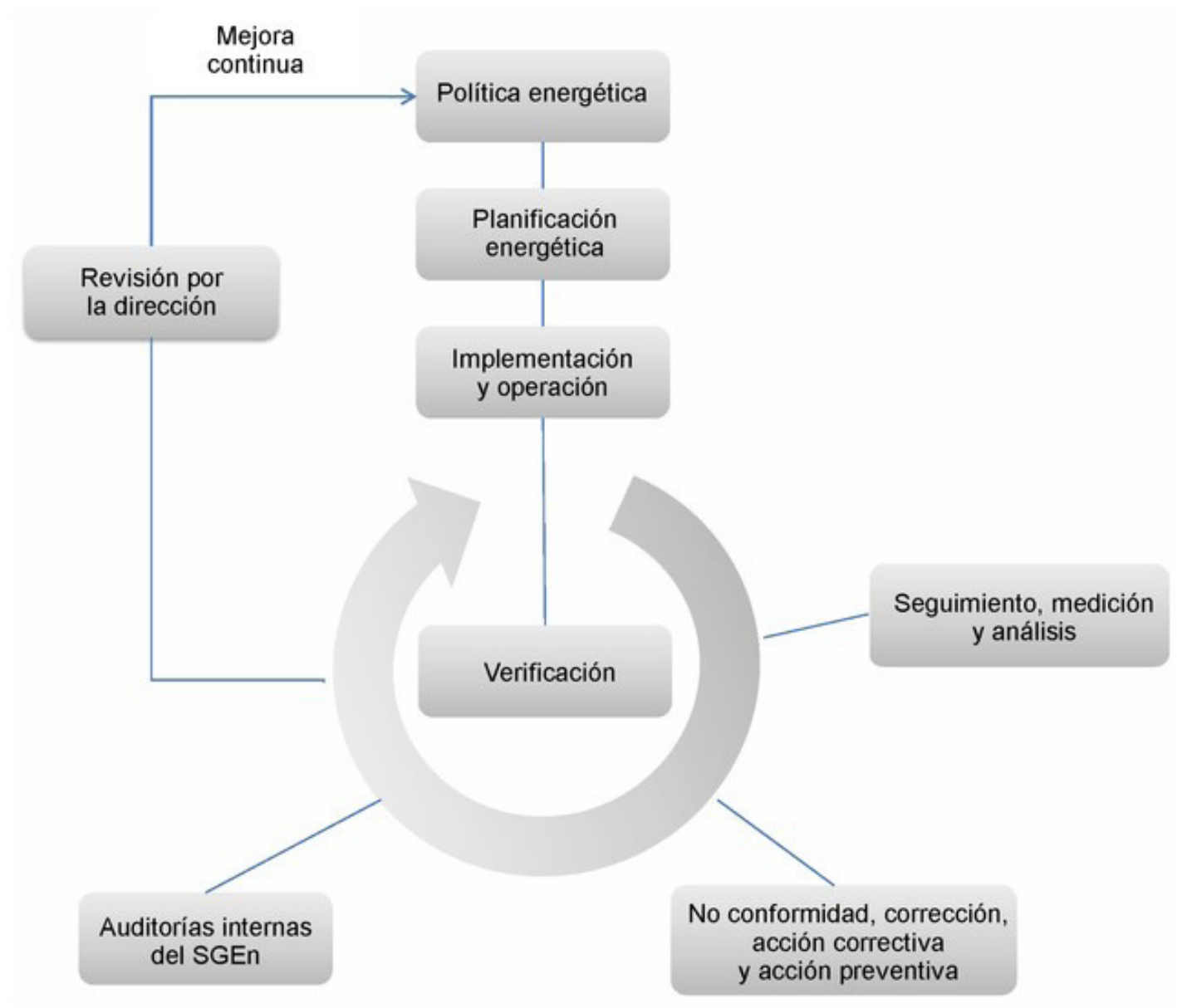

Fig. 1. Modelo de sistema de gestión de la energía ISO 50001. Fuente: [21].

La ISO 50001 está fundamentada en el enfoque "Planificar-Hacer-Verificar-Actuar" para la mejora continua, proporcionando los requisitos para la misma [16]. En este estudio se utilizó la metodología propuesta en [15]:

1. Evaluar la efectividad del sistema de control actual.

2. Proponer indicadores de desempeño (EnPI) efectivos y establecer la línea base.

3. Validar las herramientas propuestas. 
4. Implementar las herramientas desarrolladas.

5. Los indicadores de desempeño se seleccionarán en función de la correlación adecuada entre parámetros de operación. La predicción precisa del consumo energético es esencial para una gestión energética efectiva en edificaciones hospitalarias [7]. En la gestión energética se consideran que correlaciones de $\mathrm{R}^{2}>0.6$ pueden utilizarse como indicadores potenciales, mientras que correlaciones $\mathrm{R}^{2}>0.8$ son indicadores potenciales fuertes [15], [17]-[20].

En el paso 4 de la metodología se implementarán la herramienta de gestión desarrollada para la clínica, como se muestra en la Fig. 2.

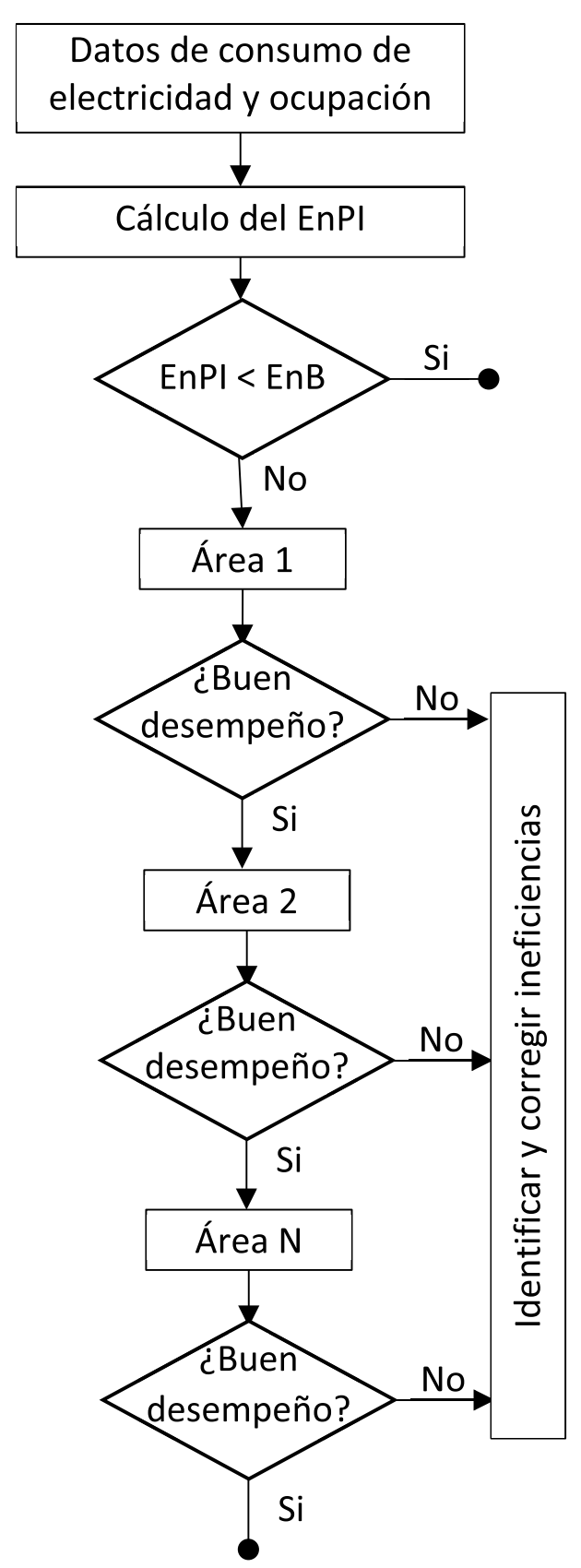

Fig. 2. Metodología de implementación de la gestión energética.

Fuente: Autores.

La metodología de gestión compara el EnPI definido para la clínica con la línea base determinada con los datos de consumo de la clínica. Si se detectan ineficiencias se realiza una campaña de detección jerarquizando las áreas por su consumo eléctrico. En el proceso de mejora continua se recomienda revisar el EnPI cada 2 años para actualizarlo.

\section{A. Clínica Portoazul}

El complejo Portoazul, ubicado en el municipio de Puerto Colombia (Atlántico, Colombia), se enfoca en la prestación de servicios de salud a la ciudad de Barranquilla, y la región Caribe colombiana en general.

El complejo incluye un edificio dedicado a la clínica, un edificio de apoyo con servicios de imágenes diagnósticas y un edificio de consultorios, además de la sección de parqueos y áreas técnicas.

El complejo ofrece un concepto de atención médica integral, segura y humanizada basada en el trabajo conjunto e innovación de la práctica médica. Esta atención requiere un soporte tecnológico de equipos de última tecnología con determinados consumos de energía. Las tres 
torres del complejo se interrelacionan operativa y funcionalmente. En la torre de consultorios realizan consultas la mayoría de los médicos especialistas adscritos. Una torre ofrece todos los servicios de imágenes diagnósticas, la última es la Clínica Portoazul. La clínica tiene un área de $19380 \mathrm{~m}^{2}$ distribuidos en 9 pisos y una cubierta donde se ubican las áreas técnicas de apoyo. Los servicios ofrecidos por la clínica se muestran en la Tabla 1.

Tabla 1. Servicios que ofrece la clínica Portoazul

\begin{tabular}{|c|c|}
\hline Servicio & Características \\
\hline UCI adultos & 18 camas \\
\hline UCI pediátricas & 7 camas \\
\hline UCI neonatales & 12 camas \\
\hline Quirófanos de cirugía & 7 salas \\
\hline Quirófanos de maternidad & 2 salas \\
\hline Urgencias & $\begin{array}{l}8 \text { consultorios } \\
12 \text { boxes de atención }\end{array}$ \\
\hline Zona de recuperación & 28 camas \\
\hline $\begin{array}{l}\text { Zonas de reanimación (divididos por especialidades } \\
\text { entre adultos y pediátricos) }\end{array}$ & 2 \\
\hline Imágenes diagnósticas & $\begin{array}{l}2 \text { rayos } \mathrm{x} \\
1 \text { tomógrafo } \\
1 \text { ecografía }\end{array}$ \\
\hline Habitaciones de hospitalización & $25 \mathrm{~m} 2$ \\
\hline Diagnóstico cardiológico & Consultorios VIP \\
\hline Unidad de oncología & 1 \\
\hline Servicios de rehabilitación & 1 \\
\hline Laboratorio clínico y sede de toma de muestras & Torre médica \\
\hline Esterilización & 1 \\
\hline Central de mezclas & 1 \\
\hline Cocina (servicio de restaurante) & 1 \\
\hline Áreas de apoyo al público (ej. restaurante 3er piso) & 1 \\
\hline Cafés en salas de espera de hospitalización & 1 \\
\hline Salas de espera & 6 \\
\hline Auditorio para 60 personas & 1 \\
\hline Ascensores & $\begin{array}{l}3 \text { de servicio de camillas } \\
3 \text { de uso público }\end{array}$ \\
\hline
\end{tabular}

Fuente: Autores

Para evitar la influencia de valores atípicos en el desarrollo de la línea base en el análisis estadístico se usa el filtrado de Hampel [22], que aplica la mediana de la desviación absoluta MAD (1):

$$
\mathrm{MAD}=1.4826 \cdot \mathrm{Mk}
$$

Siendo (2):

$$
\mathrm{Mk}=\operatorname{mediana}\left\{\mathrm{X} 1-\mathrm{X}^{*} ; \mathrm{X} 2-\mathrm{X}^{*} ; \ldots ; \mathrm{Xn}-\mathrm{X}^{*}\right\}
$$

Donde:

$$
\begin{array}{ll}
\mathrm{n} & : \text { Número de datos } \\
\mathrm{X}_{1,2,3, \ldots, \mathrm{n}} & : \text { Datos } \\
\mathrm{X}^{*} & : \text { mediana }=\left\{X_{1}, X_{2}, \ldots, X_{\mathrm{n}}\right\}
\end{array}
$$

De esta forma se filtran los datos fuera de rango $(X<(\bar{X}-\mathrm{MAD}, \bar{X})>X>(\bar{X}+\mathrm{MAD}))$ que son identificados como valores atípicos. 


\section{Resultados y Discusión}

El diagnóstico energético de la clínica se desarrolló con datos recopilados entre el 1 de mayo de 2018 y el 31 de enero de 2019 (usando equipos de medición del sistema de control de la clínica y un analizador de redes Fluke 435 series 6). Se seleccionó este periodo por la instalación en mayo del 2018 de medidores de consumo eléctrico para diferentes áreas de la clínica, permitiendo una medición más completa de la clínica. La Fig. 3 muestra el consumo de portadores energéticos para la Clínica Portoazul entre mayo de 2018 y enero de 2019.

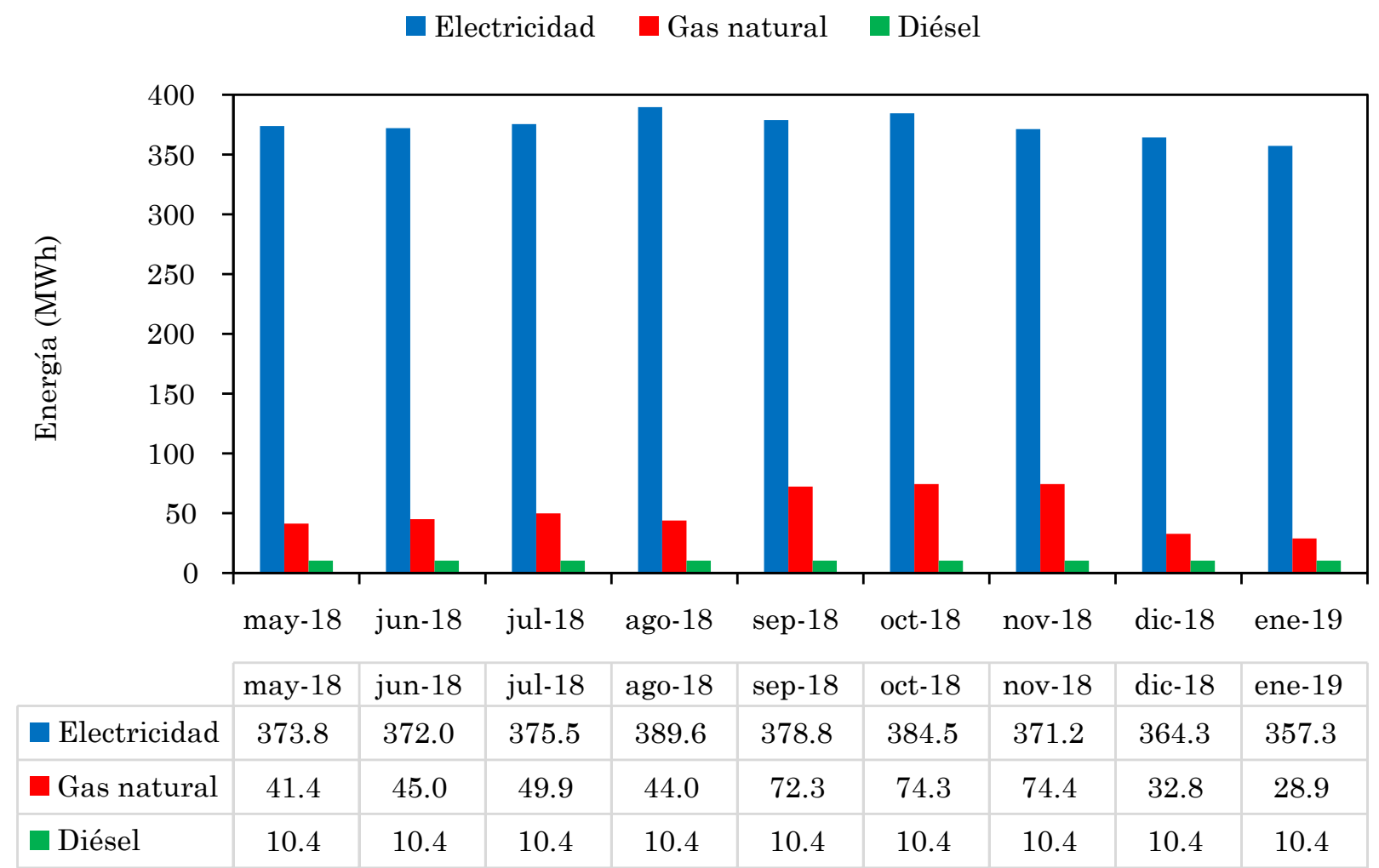

Fig. 3. Consumo de portadores energéticos en la Clínica Portoazul. Fuente: Autores.

En la Fig. 3, la electricidad representa el 86\% del consumo de energía de la clínica, a un costo de 1192 millones de COP; seguida del gas natural (12\%) a un costo de 80 millones de COP; y el diésel (2\%), a un costo de 182 millones de COP.

La demanda de potencia de la clínica durante el día se puede observar en la Fig. 4.

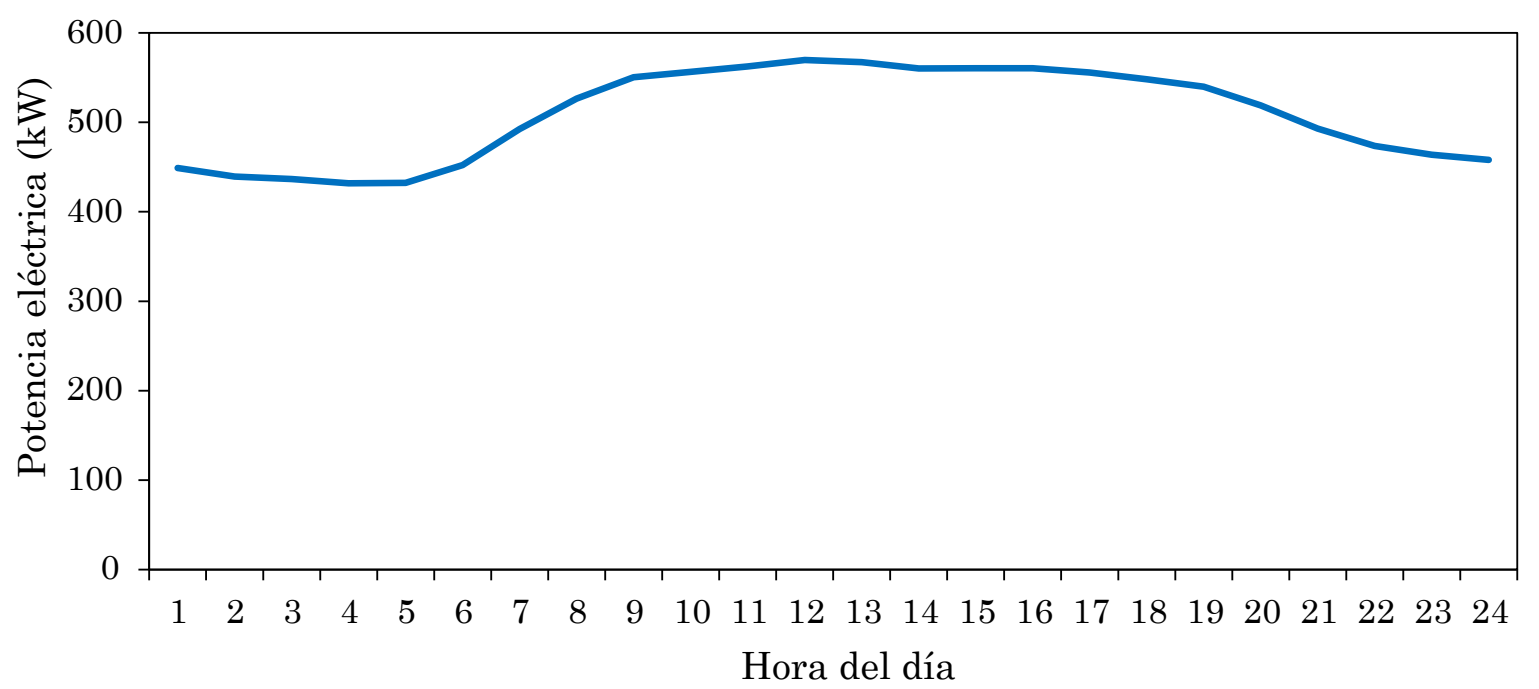

Fig. 4. Demanda de potencia promedio en la Clínica Portoazul. Fuente: Autores.

La medición muestra que la mayor demanda de potencia se observa durante el día, entre las 9 y las 19 horas. En general, la potencia varía entre 400 y $600 \mathrm{~kW}$.

El consumo de electricidad mensual de la clínica, entre el 1 de mayo de 2018 y el 31 de enero de 2019, se indica en la Fig. 5. En este caso, se presentan las mediciones diarias del consumo energético de la clínica. 


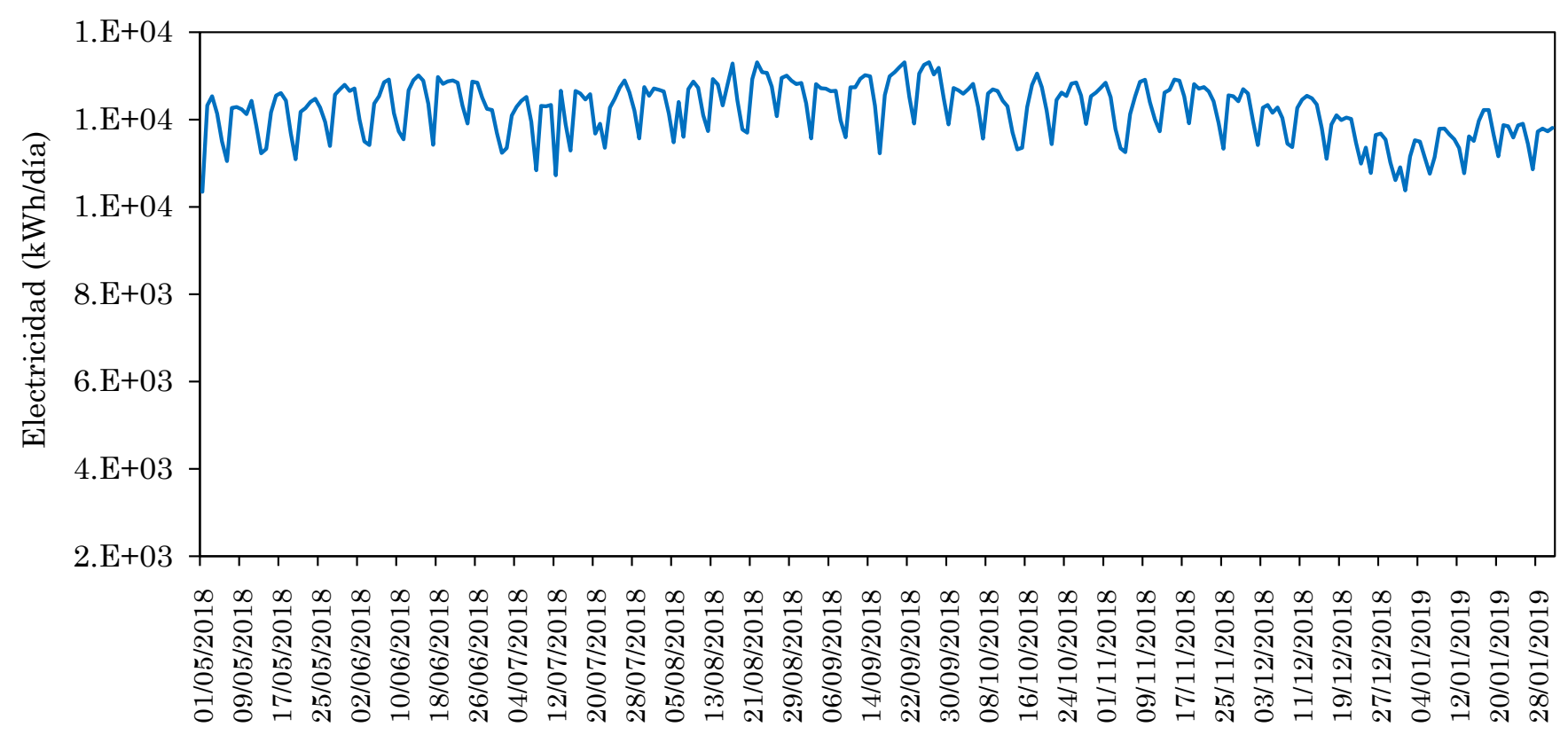

Fig. 5. Consumo de electricidad de la clínica Portoazul. Fuente: Autores.

En general, el consumo eléctrico de la clínica se mantiene estable entre 10000 y 14000 kWh por día.

La distribución del consumo eléctrico por sistemas de la clínica se refleja en la Fig. 6. En este caso se evidencia el consumo diario de las áreas medidas durante el estudio.

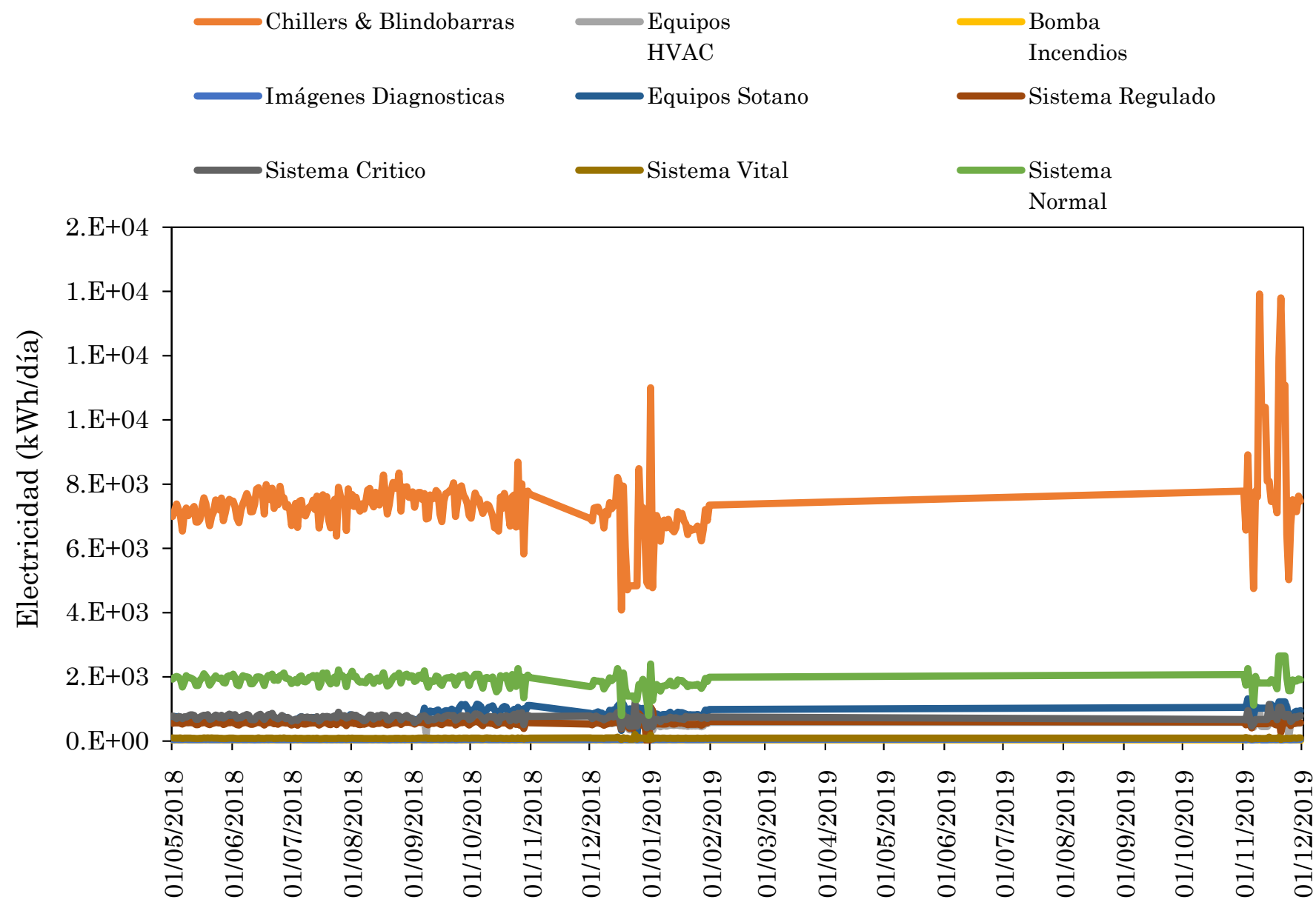

Fig. 6. Consumo de electricidad de la clínica Portoazul. Fuente: Autores.

En total, se consideran nueve subsistemas en el sistema eléctrico de la clínica. Las mayores demandas de electricidad se localizan en los Chillers \& Blindobarras y en el Sistema Normal representando el $65 \%$ de la carga de total (Fig. 6).

En la Fig. 7 se muestra el comportamiento del chiller (enfriador) en diferentes horarios del día:

- 7:30 a.m. - 11:30 a.m.

- 11:30 a.m. - 3:30 p.m.

- 3:30 p.m. - 7:30 p.m.

- 7:30 p.m. - 7:30 a.m. 


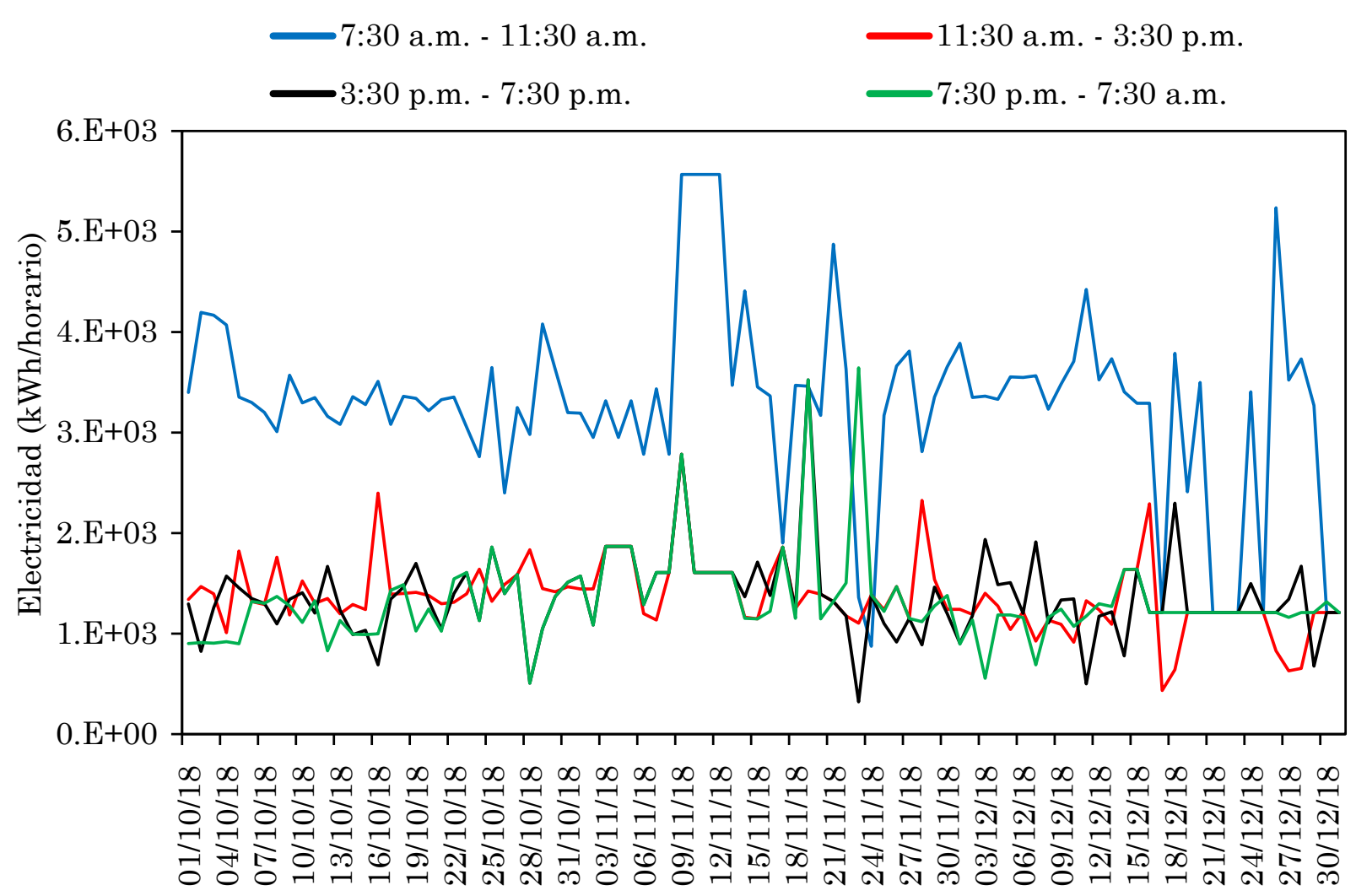

Fig. 7. Consumo de electricidad de los chillers Fuente: Autores.

Los resultados demuestran como el mayor consumo eléctrico ocurre entre las 7:30 a.m. y las 11:30 a.m., en el resto del día el consumo se mantiene relativamente estable. Mientras que durante la noche se registra el menor consumo.

En la Fig. 8 se evidencia el comportamiento de la ocupación y el consumo de electricidad diario para el periodo seleccionado.

Para determinar la influencia de la actividad de la clínica sobre el consumo de electricidad, se desarrolla un análisis de correlación entre el consumo eléctrico y la ocupación (número de pacientes). La Fig. 8 muestra la relación entre la ocupación y el consumo eléctrico de la clínica.

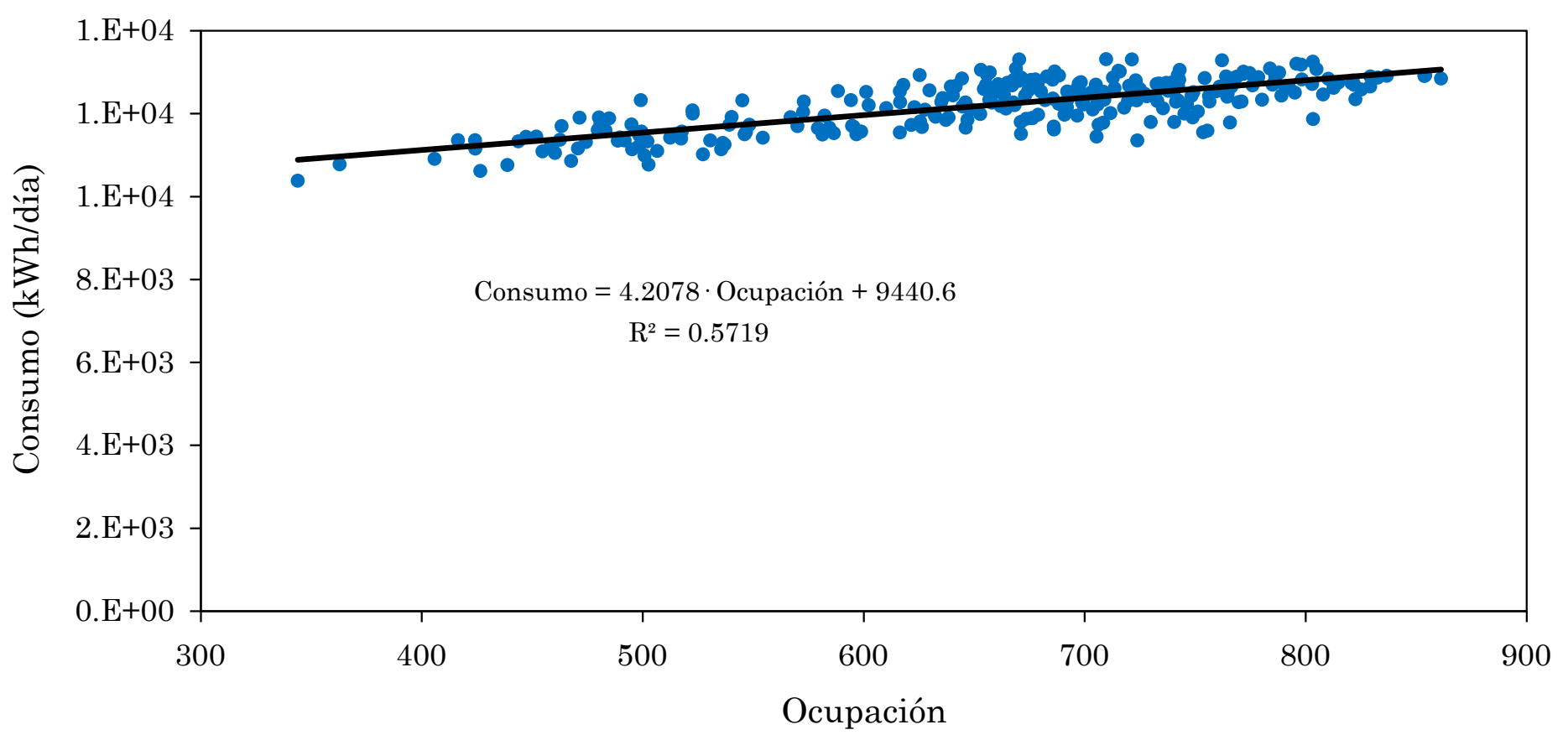

Fig. 8. Relación ocupación equivalente y consumo eléctrico. Fuente: Autores.

Como resultado se obtiene una correlación menor a 0.6, valor mínimo recomendado para considerar la correlación como un indicador potencial [15], [17]-[20]. Por consiguiente, se desarrolla un indicador equivalente según lo recomendado por la ISO 50006 [23]. En este caso, la ocupación equivalente se entiende como la suma de los pacientes, el personal técnico y administrativo, y el personal de asistencia (médicos y enfermeras). La Fig. 9 muestra la correlación entre la ocupación equivalente y el consumo eléctrico. 


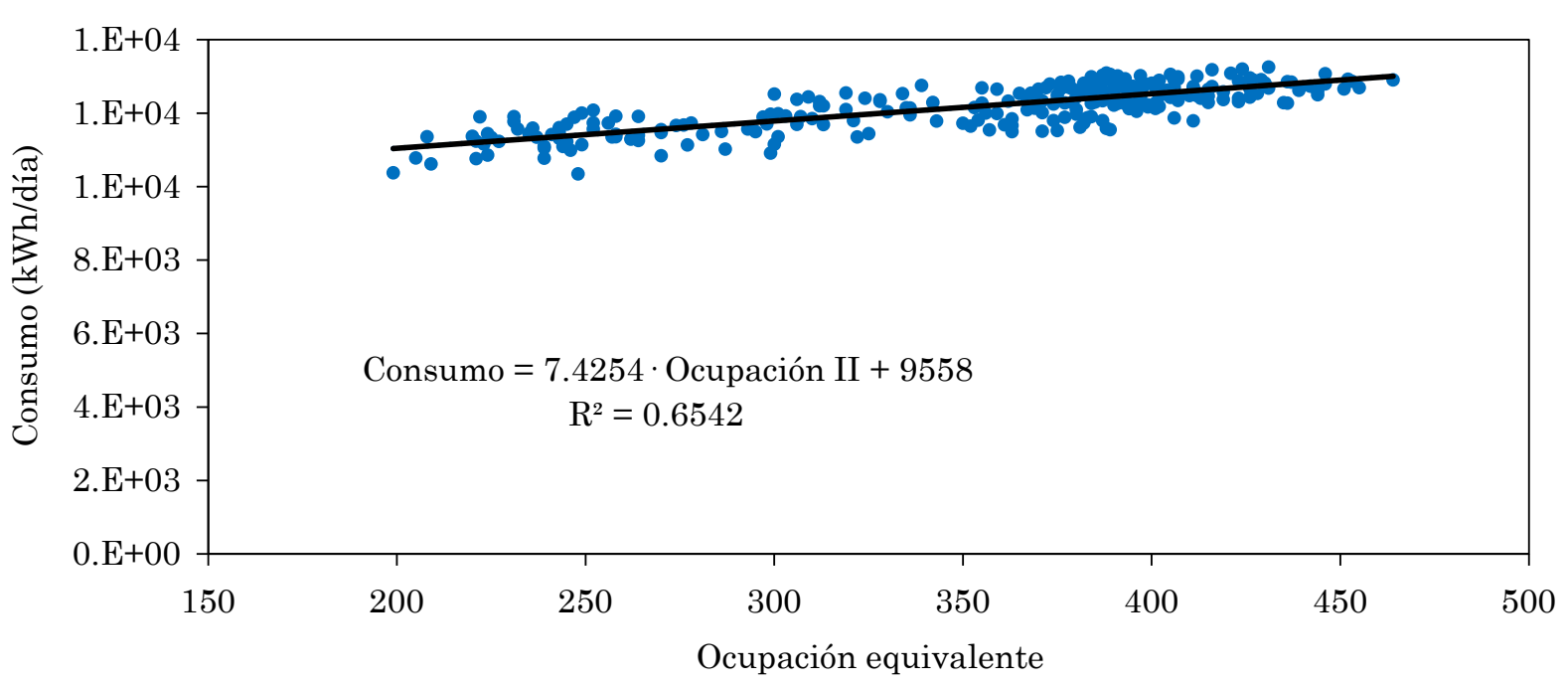

Fig. 9. Relación ocupación equivalente y consumo eléctrico. Fuente: Autores.

La Fig. 9 muestra como la ocupación equivalente tiene una correlación adecuada para su uso como un indicador potencial para evaluar el desempeño energético de la clínica $\left(R^{2}>0.6\right)$ [15], [17]-[20].

En este caso, la línea base sería (3):

$$
\text { EnB }=7.4254 \cdot \text { Ocupación II + } 9558
$$

El EnPI se determina a partir de (3) [15] y se implementó entre enero y febrero de 2020:

$$
\text { EnPI }=\frac{\text { Consumo }}{\text { Ocupación equivalente }}
$$

La Fig. 10 muestra la línea meta utilizada como gráfico de control de la clínica, y los resultados de la implementación del EnPI.

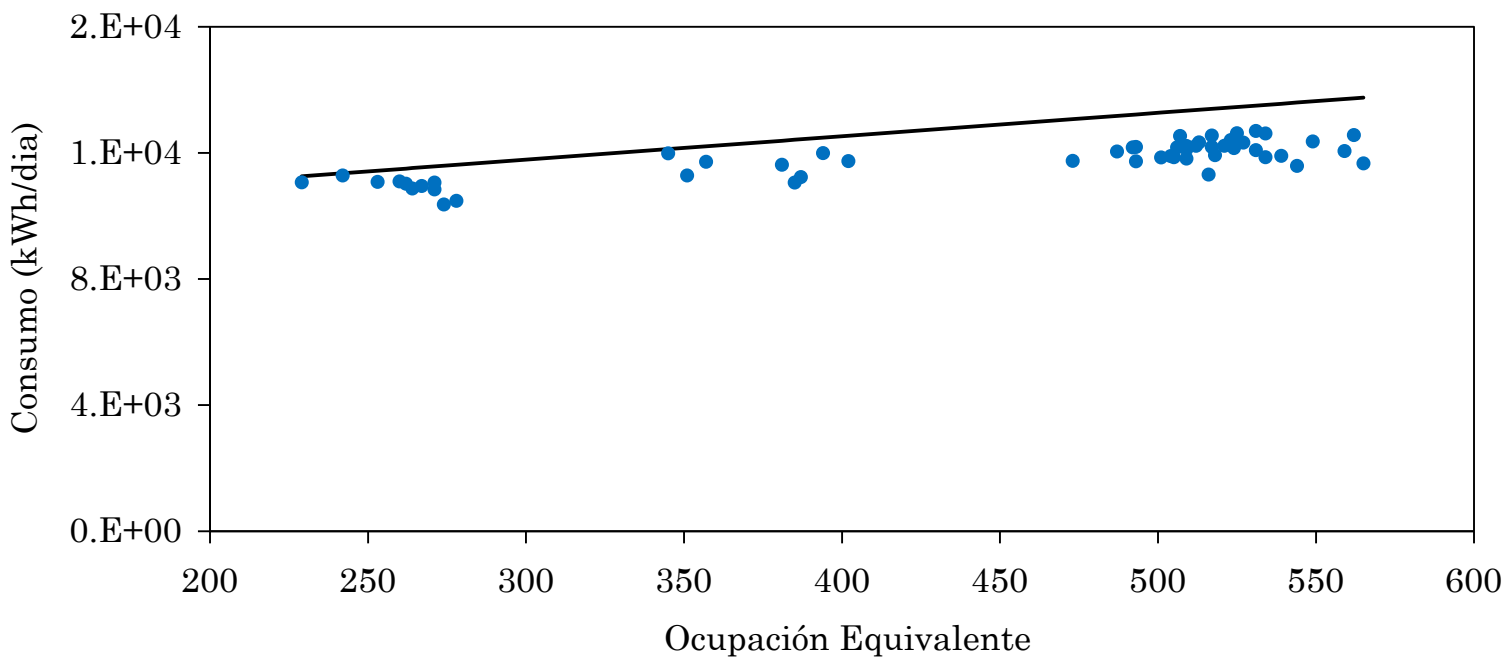

Fig. 10. Línea meta y consumos de electricidad (enero-febrero2020). Fuente: Autores.

Dado el peso de los chillers en el consumo total de la clínica, se desarrolló un sistema de monitoreo y control individual. En este caso se desarrolló un Sistema de Administración de Sistemas de Climatización (SASC) para monitorear de forma constante su desempeño energético. El sistema incluye el monitoreo continuo de parámetros clave del desempeño energético, que comprende:

- Presiones y temperaturas del refrigerante.

- Consumo de potencia en los compresores.

- Temperatura de agua en el evaporador.

- Temperaturas de agua y/o aire en el condensador. 
Estos parámetros se usan para evaluar:

- Índices: COP, kW/TR, SEI.

- Consumo energético.

- Horas de funcionamiento, paradas, disponibilidad.

- Causas posibles de paradas.

- Costo de energía en horas de funcionamiento.

En este caso se programan alarmas cuando los parámetros se salen de su rango de operación recomendado por el fabricante.

Adicionalmente, se usa la línea meta como gráfico de control para monitorear el desempeño energético de la clínica. Es decir, para identificar cuando, a existe un problema en la demanda de frío a pesar de que los chillers están funcionando correctamente (ej. las manejadoras de aire de algún área desocupada o con poco personal están operando al 100\% de su capacidad generando una demanda de frío mayor a la real).

La Fig. 10 muestra que el uso del indicador permitió monitorear y controlar el consumo de electricidad en la clínica. Durante este periodo, el consumo diario de electricidad se redujo entre un $1 \%$ y un $15 \%$ comparado con la línea meta. Adicionalmente, se reduce el costo de la electricidad asociado al consumo eléctrico.

En este caso se implementó la metodología descrita en la Fig. 2, revisando jerárquicamente las áreas una vez se detectan ineficiencias.

\section{Discusión}

El sistema de monitoreo del consumo eléctrico diario de la clínica, basado en la Fig. 9, permitió detectar comportamientos anómalos e ineficiencias. Se tomaron medidas específicas en el sistema de climatización para monitorear el desempeño de los chillers.

Así, se establecen alarmas cuando un parámetro se sale de su rango de operación afectando el desempeño energético del sistema. En estudios posteriores, puede tomarse el sistema de gestión como base para definir el período óptimo ejecución de mantenimientos preventivos.

El EnPI desarrollado para la clínica no es complejo de calcular e implementar, por lo que puede ser manejado por el personal técnico, lo cual es esencial para el éxito del sistema de gestión. Comparado con otros estudios [6], [7], [9], [10], este es un enfoque más práctico y económico que no requiere la introducción de nuevas tecnologías.

\section{Conclusiones}

El EnPI desarrollado es manejable para el personal de la clínica, por lo que se integró de forma efectiva en la gestión diaria, y podría adaptarse a otras instalaciones hospitalarias. En general, la implementación del sistema de gestión contribuyó reducir el consumo diario entre un $1 \%$ y un $15 \%$ comparado con la línea base. Adicionalmente, se reducen los costos económicos de la electricidad entre el $1 \%$ y el $15 \%$ en el período monitoreado.

\section{REFERENCIAS}

[1] IEA \& UN, 2019 Global status report for buildings and construction, LD, UK: GlobalABC, 2019. Available: https://www.worldgbc.org/news-media/2019-global-status-report-buildings-and-construction

[2] L. Chen, Q. Xu, Y. Yang \& J. Song, "Optimal energy management of smart building for peak shaving considering multi-energy flexibility measures," Energy Build, vol. 241, no. 3, pp. 1-19, Mar. 2021. https://doi.org/10.1016/j.enbuild.2021.110932

[3] D. Rendón, "Colombia en su camino a ser más eficiente energéticamente", Forbes Colombia, Mar. 6, 2020. Available: https://forbes.co/2020/03/06/actualidad/colombia-en-su-camino-a-ser-mas-eficienteenergeticamente/

[4] A. Teke \& O. Timur, "Assessing the energy efficiency improvement potentials of HVAC systems considering economic and environmental aspects at the hospitals," Renew Sustain Energy Rev, vol. 33, pp. 224-235, May. 2014. https://doi.org/10.1016/j.rser.2014.02.002

[5] N. Thinate, W. Wongsapai \& D. Damrongsak, "Energy Performance Study in Thailand Hospital Building," Energy Procedia, vol. 141, pp. 255-259, Dec. 2017. https://doi.org/10.1016/j.egypro.2017.11.102 
[6] M. G. Gomes, A. M. Rodrigues \& F. Natividade, "Thermal and energy performance of medical offices of a heritage hospital building," J. Build Eng, vol. 40, pp. 1-19, Aug. 2021. https://doi.org/10.1016/j. jobe.2021.102349

[7] L. Cao, Y. Li, J. Zhang, Y. Jiang, Y. Han \& J. Wei, "Electrical load prediction of healthcare buildings through single and ensemble learning," Energy Reports, vol. 6, pp. 2751-2767, Nov. 2020. https://doi. org/10.1016/j.egyr.2020.10.005

[8] A. Patiño, "Technology trends for business productivity increase," INGE CUC, vol. 11, no. 2, pp. 84-96, Jul. 2015. https://doi.org/10.17981/ingecuc.11.2.2015.09

[9] A. Buonomano, F. Calise, G. Ferruzzi \& A. Palombo, "Dynamic energy performance analysis: Case study for energy efficiency retrofits of hospital buildings," Energy, vol. 78, pp. 555-572, Dec. 2014. https://doi. org/10.1016/j.energy.2014.10.042

[10] M. Prada, I. F. Pradab, M. Cristeab, D. E. Popescuc, C. Bungăud, L. Aleyae \& C. C. Bungăuab, "New solutions to reduce greenhouse gas emissions through energy efficiency of buildings of special importance Hospitals," Sci Total Environ, vol. 718, p. 1-16, May. 2020. https://doi.org/10.1016/j.scitotenv.2020.137446

[11] B. Nourdine \& A. Saad, "About energy efficiency in Moroccan health care buildings," Mater Today Proc, vol. 39, pp. 1141-1147, Apr. 2020. https://doi.org/10.1016/j.matpr.2020.04.135

[12] MME \& UPME, Plan de acción indicativo de eficiencia energética 2017 - 2022, Bog. Col., Minminas, 2016. Disponible en https://www1.upme.gov.co/Paginas/Plan-de-Acci\%C3\%B3n-Indicativo-de-EficienciaEnerg\%C3\%A9tica-PAI-PROURE-2017---2022.aspx

[13] J. Liu, Q. Zhanga, Z. Dong, X. Li, G. Li, Y. Xi \& K. Li, "Quantitative evaluation of the building energy performance based on short-term energy predictions," Energy, vol. 223, p. 120065, May. 2021. https://doi. org/10.1016/j.energy.2021.120065

[14] A. McKane, D. Desai, M. Matteini, W. Meffert, R. Williams \& R. Risse, "Thinking Globally : How ISO 50001 - Energy Management can make industrial energy efficiency standard practice," EETD, CA, USA, Technical Report, 2010. https://doi.org/10.2172/983191

[15] J. J. Cabello, V. Sousa, A. Sagastume, M. A. Guerra-Plasencia, D. Haeseldonckx \& C. Vandecasteele, "Tools to improve forecasting and control of the electricity consumption in hotels," J Clean Prod, vol. 137, pp. 803-812, 2016. https://doi.org/10.1016/j.jclepro.2016.07.192

[16] Energy management systems, ISO 50001:2018, International Organization for Standardization, ISO, GVA, CH, 2018. https://www.iso.org/standard/69426.html

[17] S. Becken, C. Frampton \& D. Simmons, "Energy consumption patterns in the accommodation sector The New Zealand case," Ecol. Econ., vol. 39, no. 3, pp. 371-386, Dec. 2001. https://doi.org/10.1016/S09218009(01)00229-4

[18] S. Deng, "Energy and water uses and their performance explanatory indicators in hotels in Hong Kong," Energy Build., vol. 35, no. 8, pp. 775-784, Sep. 2003. https://doi.org/10.1016/S0378-7788(02)00238-4

[19] N. E. Matson \& M. A. Piette, "Review of California and national methods for energy-performance benchmarking of commercial buildings," LBNL, CA, USA, Report Technical LBNL-57364, 2005. https://doi. org/10.2172/887197

[20] P. Bohdanowicz and I. Martinac, "Determinants and benchmarking of resource consumption in hotelsCase study of Hilton International and Scandic in Europe," Energy Build., vol. 39, no. 1, pp. 82-95, Jan. 2007. https://doi.org/10.1016/j.enbuild.2006.05.005

[21] Sistemas de gestión de la energía, ISO 50001:2011(es), International Organization for Standardization, ISO, GVA, CH, 2018. Available: https://www.iso.org/obp/ui/\#iso:std:iso:50001:ed-1:v1:es

[22] J. J. Cabello, A. Sagastume, V. Sousa, H. Hernández, M. Balbis, J. Silva, E. M. Noriega \& C. Vandecasteele, "Energy management in the formation of light, starter, and ignition lead-acid batteries," Energy Effic, vol. 12, no. 5, pp. 1219-1236, 2019. https://doi.org/10.1007/s12053-018-9741-6

[23] Energy management systems - EnB and EnPI, ISO 50006, International Organization for Standardization, ISO, GVA, CH, 2014. Available: https://www.iso.org/standard/51869.html

Sebastián Andrés Botero Ebrat. Nivel de formación: Magister. Afiliación institucional: Clínica Portoazul (Barranquilla, Colombia). 